\title{
A retrospective study of the initial 25 COVID-19 patients in Luoyang, China
}

\author{
Xiaopei Duan $^{1}$ (D) Xinyu Guo ${ }^{2} \cdot$ Jun $^{\text {Qiang }}{ }^{1}$
}

Received: 23 February 2020 / Accepted: 29 April 2020 / Published online: 26 May 2020

(c) Japan Radiological Society 2020

\begin{abstract}
Purpose To summarize the chest CT imaging and clinical features of the initial COVID-19 patients and provide a clinical diagnostic method that is more effective and can be performed earlier.

Methods This retrospective study investigated the clinical, laboratory and imaging information of 25 patients in the Luoyang area. There were $15(60 \%)$ male and $10(40 \%)$ female patients ranging from 24 to 88 years old (52 \pm 19.30$)$. Data were analyzed by Microsoft Excel and are expressed as the mean \pm standard deviation or percentage.

Results Thirteen (52\%) patients had been in Wuhan or were in contact with people who had been in Wuhan, and ten (40\%) patients were infected by their families or colleagues. The median time from initial symptoms to diagnosis was 7 days. Ninety-two percent of patients had respiratory symptoms, and $8 \%$ of them had digestive symptoms. Fever (92\%), cough $(60 \%)$ and fatigue (56\%) were the most common symptoms. Most patients had a normal or reduced WBC (96\%), reduced lymphocyte count (60\%), increased CRP (48\%) and increased ESR (44\%). Ground glass opacity (GGO) was the typical radiological finding on chest $\mathrm{CT}$.
\end{abstract}

Conclusion Characteristic chest CT imaging features could appear earlier than the viral nucleic acid assay results.

Keywords SARS-CoV-2 $\cdot$ COVID-19 pneumonia $\cdot$ CT

\section{Introduction}

In December 2019, a cluster of patients with unknown pneumonia was reported in Wuhan, Hubei Province, China [1-3]. After the epidemiological survey and genome sequencing analysis, a novel coronavirus was identified [4]. The International Committee on Taxonomy of Viruses (ICTV) announced it as severe acute respiratory syndrome coronavirus 2 (SARS-CoV-2) [5], and the World Health Organization (WHO) announced that the disease caused by SARSCoV-2 was coronavirus disease 2019 (COVID-19). Within

Xiaopei Duan and Xinyu Gu contributed equally to the article.

Jun Qiang

15838815301@163.com

1 Department of Radiology, The First Affiliated Hospital, College of Clinical Medicine, Medical College of Henan University of Science and Technology, Luoyang 471003, China

2 State Key Laboratory of Respiratory Disease, Sino-French Hoffmann Institute, School of Basic Medical Science, Guangzhou Medical University, Guangzhou 511436, China
2 months, it had spread to different areas and countries. As of February 8, 2020, more than 30,000 cases of laboratoryconfirmed SARS-CoV-2 infection were reported around the world, including 25 confirmed cases in the area of Luoyang, Henan Province, China. SARS-CoV-2 can cause severe respiratory illness, similar to SARS and Middle East respiratory syndrome (MERS), and has been found to be adept at human-to-human transmission [2, 6]. China launched an emergency response to the outbreak, and the WHO also declared it a public health emergency of international concern (PHEIC). In the case of SARS-CoV-2, there have been some clues suggesting that, sometimes, an individual with highly severe SARS-CoV-2 disease will only cause a few infections; conversely, individuals with a moderate disease or latent infection can occasionally cause many infections [7].

As a result, early detection, isolation and treatment are the key steps to preventing further spread of this disease. According to the fifth version of the Diagnosis and Treatment of pneumonitis caused by the new coronavirus published by the National Health Commission of the People's Republic of China, the SARS-CoV-2 laboratory-confirmation method, 
which involves viral nucleic acid detection by real-time reverse-transcriptase polymerase-chain-reaction (PCR) assay using nasal and pharyngeal swab specimens, has shown false negatives, and the detectability is still limited. Thus, CT imaging plays an important role in the diagnosis of SARS-CoV-2 pneumonia.

Given the concept of the early diagnosis and treatment of SARS-CoV-2, this article mainly focused on the 25 initial laboratory-confirmed patients in the Luoyang area, discussing their imaging features and clinical characteristics.

\section{Materials and methods}

\section{Patients}

From January 10 to February 8, 2020, 25 patients with laboratory-confirmed SARS-CoV-2 infection in the area of Luoyang, Henan Province, China, were enrolled in the study. Patients were diagnosed with COVID-19 based on the WHO interim guidance [8]. A patient confirmed to have SARSCoV-2 was defined as a positive case according to a PCR assay using nasal and pharyngeal swab specimens [1]. PCR results were obtained after a 1- to 3-day delay and were then used to identify confirmed cases. Of the 25 patients, three had severe conditions, and the others had moderate conditions. Ten patients underwent follow-up CT, and the lung lesions of two people improved, whereas eight of them gradually absorbed after experiencing consolidation changing. Patients were divided into three groups according to the CT scanning timing after the onset of patients' symptoms: group $1, \leq 7$ days; group 2, 8-14 days; and group 3, 14-21 days. Two of the 25 patients were cured and discharged.

The Ethics Committee of the First Affiliated Hospital, College of Clinical Medicine, Medical College of Henan University of Science and Technology approved this retrospective study. The requirement for informed patient consent was waived by the ethics committee for this retrospective study.

\section{Clinical data collection}

The epidemiological characteristics (including recent exposure and history), clinical symptoms and signs, laboratory examination and radiologic data were extracted from the electronic medical records of the First Affiliated Hospital of Henan University of Science and Technology. Laboratory data consisted of hematology, urinalysis, blood chemistry, blood immunology, coagulation tests, and other routine examinations. Radiologic data included digital radiography (DR) and computed tomography (CT). PCR assays were performed by the Center for Disease Control (CDC) in Henan, China.
All data were collected by using Microsoft Excel. Data are expressed as the mean \pm standard deviation or percentage.

\section{Image acquisition}

Some patients underwent DR examination of the chest using a Philips (Digtal Diagnost) DR. All patients underwent CT scans of the chest using a Philips Brilliance (Philips Medical System, Andover, Massachusetts) 16-section MDCT $(120 \mathrm{kV}, 220 \mathrm{mAs}, 0.75-\mathrm{mm}$ section thickness, pitch of 1.2, $16 \times 0.75$ detector configuration). Reconstruction was performed using the CT Viewer application of the EBW workstation (Philips Extended Brilliance Workspace, Philips Medical). Two radiologists (JQ with 19 years of experience and XD with 15 years of experience, both generalists without fellowship training who resolved discrepancies by consensus) independently and retrospectively evaluated all the images on a digital EBW workstation. Radiological features were defined according to the Glossary of Terms established by the Fleischner Society.

\section{Results}

\section{Basic information}

As shown in Table 1, there were $15(60 \%)$ male and $10(40 \%)$ female patients ranging from 24 to 88 years old $(52 \pm 19.30)$. Nine patients $(40.90 \%)$ had gone to Wuhan or had a history of contact with someone who had been in Wuhan, 13 (54.12\%) patients had a history of contact with confirmed SARS-CoV-2 patients or family cluster phenomena, and the remaining two people had unknown causes of infection. Twelve patients (48\%) had comorbidities, including diabetes (20\%), hypertension $(20 \%)$, cardiac disease $(8 \%)$, hepatic adipose infiltration (4\%), coronary disease (4\%) and syphilis (4\%). Of these patients, no children or adolescents were infected.

\section{Clinical characteristics}

The median latent period was 9 days (range, 4-21). The median time from initial symptoms to PCR diagnosis was 7 days (range, $0-13$ ).

All patients had symptoms before diagnosis, $92 \%$ had respiratory symptoms and $8 \%$ had digestive symptoms. As shown in Table 1, fever (92\%), cough (60\%) and fatigue (56\%) were the most common initial symptoms, and some patients had phlegm (36\%), dyspnea (28\%) and sore throat (20\%) symptoms. A small number of patients experienced loss of appetite (12\%), stuffy and runny nose (8\%), and nausea and vomiting (4\%). 
Table 1 Basic information and clinical characteristics of 25 COVID19 patients

\begin{tabular}{|c|c|}
\hline Information & Number $(\%)$ \\
\hline \multicolumn{2}{|l|}{ Characteristic } \\
\hline \multicolumn{2}{|l|}{ Sex } \\
\hline Male & $15(60 \%)$ \\
\hline Female & $10(40 \%)$ \\
\hline \multicolumn{2}{|l|}{ Age (year) } \\
\hline $24-88$ & $52 \pm 19.30$ \\
\hline \multicolumn{2}{|l|}{ Exposure } \\
\hline Wuhan contact & $13(52 \%)$ \\
\hline Family cluster/confirmed patient contact & $10(40 \%)$ \\
\hline Unknown & $2(8 \%)$ \\
\hline \multicolumn{2}{|l|}{ Comorbidity } \\
\hline Total & $12(48 \%)$ \\
\hline Diabetes & $5(20 \%)$ \\
\hline Hypertension & $5(20 \%)$ \\
\hline Cardiac disease & $2(8 \%)$ \\
\hline Hepatic adipose infiltration & $1(4 \%)$ \\
\hline Coronary disease & $1(4 \%)$ \\
\hline Chronic obstructive pulmonary disease & $1(4 \%)$ \\
\hline Syphilis & $1(4 \%)$ \\
\hline \multicolumn{2}{|l|}{ Latent period } \\
\hline 4-21 days & $8.90 \pm 4.95$ days \\
\hline \multicolumn{2}{|l|}{ Diagnosis time } \\
\hline $0-13$ days & $6.64 \pm 3.82$ days \\
\hline \multicolumn{2}{|l|}{ From initial symptoms } \\
\hline \multicolumn{2}{|l|}{ Initial symptoms } \\
\hline Fever $\left(38.1 \pm 0.72{ }^{\circ} \mathrm{C}\right)$ & $23(92 \%)$ \\
\hline Cough & $15(60 \%)$ \\
\hline Fatigue & $14(56 \%)$ \\
\hline Phlegm & $9(36 \%)$ \\
\hline Dyspnea & $7(28 \%)$ \\
\hline Sore throat & $5(20 \%)$ \\
\hline Loss of appetite & $3(12 \%)$ \\
\hline Stuffy and runny nose & $2(8 \%)$ \\
\hline Diarrhea & $2(8 \%)$ \\
\hline Nausea and vomiting & $1(4 \%)$ \\
\hline \multicolumn{2}{|l|}{ Laboratory examination } \\
\hline WBC, normal or reduced & $24(96 \%)$ \\
\hline Lymphocyte reduced & $15(60 \%)$ \\
\hline CRP elevated & $12(48 \%)$ \\
\hline ESR elevated & $11(44 \%)$ \\
\hline AST elevated & $4(12.67 \%)$ \\
\hline CK elevated & $1(4 \%)$ \\
\hline CK-MB elevated & $2(8 \%)$ \\
\hline
\end{tabular}

CRP C-reactive protein, ESR erythrocyte sedimentation rate, AST aspartate aminotransferase, $C K$ creatine kinase

In the early stage of COVID-19, the total white blood cell count (WBC) in the peripheral blood was normal or reduced $(96 \%)$, and the lymphocyte count $(60 \%)$ was reduced. In most patients, C-reactive protein (CRP) was elevated (48\%), and the erythrocyte sedimentation rate (ESR) was elevated (44\%). All patients had normal D-dimer levels. In $4 \%$ and $8 \%$ of patients, creatine kinase (CK) and CK-MB were elevated, respectively.

\section{Demographic and time course of diagnosis}

Ten patients (40\%) had been to Wuhan (patients 1, 2, 4, 5, 7, $11,13,14,21$, and 23 ), and two patients ( $8 \%$ ) had a history of contact with people who had been in Wuhan (patients 6 and 8 ). A total of ten patients ( $40 \%$ ) had close contact with a confirmed patient or were infected from family members (patients 9, 10, 12, 15, 18, 19, 20, 22, 24, and 25). Three patients (12\%) stated that they did not go to Wuhan or have contact with infected patients.

Characteristics of 25 laboratory-confirmed patients are shown in Fig. 1, and most patients had abnormal CT imaging results. Fifteen patients underwent PCR and CT examinations before diagnosis, which helped to diagnose the disease. In the early stage, DR had normal manifestations (patients 2, $4,5,6,8,12,14,18$, and 23), while most CT scans revealed abnormal imaging. PCR results were also negative in the early stage of the disease in patients 18, 21 and 22 .

\section{CT image findings}

According to CT imaging of patients in group 1 (Table 2), more than $50 \%$ of patients had lesions in both lungs, located in the lower region, that were pure GGO. More than three lobes were commonly involved. Group 2 showed primarily GGO with consolidation, while other lesion features were the same as those found in group 1. Group 3 showed GGO with consolidation, while other lesions were similar to those in groups 1 and 2. No pericardial or lymphadenopathy was found in our patients. All three groups had lesions mainly located in the peripheral area.

As shown in Fig. 1, in the early stage (initial symptoms within 4 days) of COVID-19, DR was normal and resulted in a lower diagnosis rate; therefore, these techniques are not recommended to be used. Figures 2, 3, 4, 5 and 6 show some typical and significant CT image findings. In the early stage, there were usually no abnormal findings on chest images or only small patchy shadows in the peripheral lung field, which could be clearly observed after the progression of the disease. In this stage, thin patchy GGO was seen on the CT, distributed along the bronchial vascular bundle, as well as thickening adjacent to the bronchial wall. As shown in Figs. 5 and 6, multiple nucleic acid assays were negative at this point, while subsequent CT manifestations revealed significant lesions 


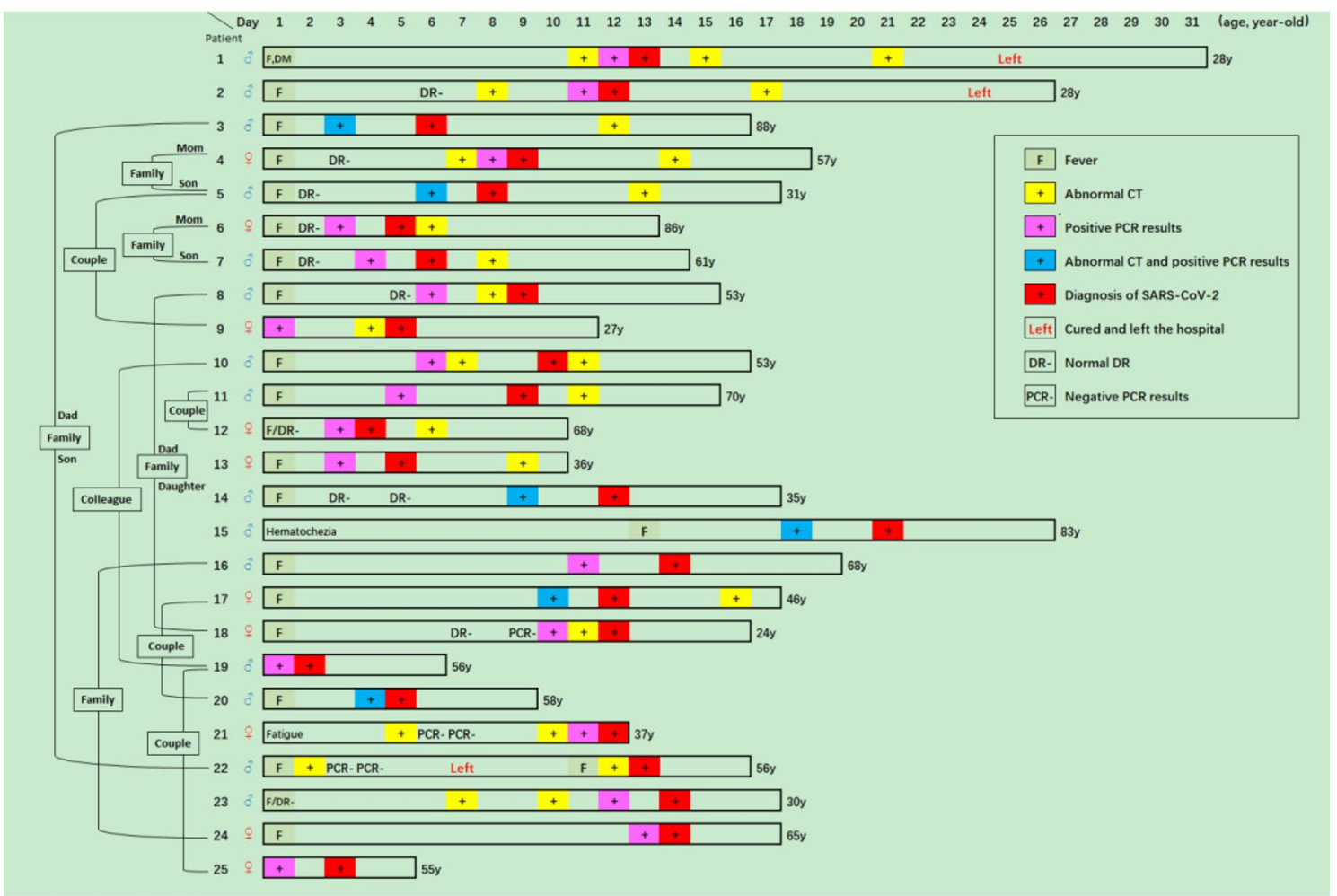

Fig. 1 Demographic and time course for diagnosing COVID-19 patients

with progression, and PCR results were also positive, reflecting the positive $\mathrm{CT}$ results for lesions at the same time. Common CT performance for bilateral lung multiple patchy ground glass opacity (GGO) and/or consolidation shadows could be accompanied by bronchial distribution of the blood vessel bundle, but the major distribution was in subpleural areas. Part of the lesion within the fine mesh shadow, halo sign or reversed halo sign could be seen in the visible edge (Fig. 4). Disease progression after the consolidation of the GGO could result in partial or complete consolidation (Fig. 3). The lesions were mainly distributed in the subpleural and oblique crack regions. Air bronchogram signs were common in consolidation shadows. The partial bronchi wall was thickened. Small blood vessels with fine reticular structures increased in the lesion. As shown in Figs. 2, 4, and 6, during the dissipation period, lesion scope and density decreased. The GGO shadow was partially or completely absorbed. A partial fibrotic stripe shadow was observed in the fusion and consolidation lesions during this period. The shadow in the lung did not completely disappear before the nucleic acid assay was negative (Fig. 2).

CT images of three patients with serious disease showed involvement of multiple lobes, even the total lobes in both lungs. The affected regions were massive and involved the central and hilum.

\section{Discussion}

Herein, we described the first diagnosis of 25 patients with COVID-19 in the Luoyang area and mainly focused on their imaging features and clinical characteristics. The Wuhan epidemic history was the essential clue. The poor immunity of the elderly and comorbidity of people contributed to SARS-CoV-2 infection. Fever (92\%), cough (60\%), and fatigue $(56 \%)$ were the most common symptoms, which was similar to other studies $[1,6,8,9]$. Most patients had normal or reduced WBC (96\%), reduced lymphocyte count $(60 \%)$, elevated CRP (48\%) and elevated ESR (44\%).

Due to familial and social cluster infection phenomena (Fig. 1), our study provided evidence of human-to-human transmission. For example, patients 10 and 19 were colleagues and had contact with confirmed COVID-19 patients in Shanghai while on business, and patient 19 transmitted the disease to his wife when he went back to Luoyang, revealing cross-city transmission. Most patients came to the hospital because of clinical symptoms (patients 3,16 and 17); as for those who had symptoms but claimed to have no Wuhan epidemiology history, determining whether they were infected with SARS-CoV-2 and establishing a clinical diagnosis was the most important issue.

GGO was the typical chest CT findings of COVID19 patients. COVID-19 started from the margins of the 
Table 2 CT features of 25 COVID-19 patients

\begin{tabular}{|c|c|c|c|}
\hline CT findings & $\begin{array}{l}\text { Group } 1(13) \\
n(\%)\end{array}$ & $\begin{array}{l}\text { Group } 2(16) \\
n(\%)\end{array}$ & $\begin{array}{l}\text { Group } 3(3) \\
n(\%)\end{array}$ \\
\hline Unilateral lung & $4(30.7)$ & $1(6.3)$ & $0(0.0)$ \\
\hline Bilateral lungs & $9(69.2)$ & $15(93.7)$ & $3(100.0)$ \\
\hline One lobe & $4(30.7)$ & $1(6.3)$ & $0(0.0)$ \\
\hline Two lobes & $4(30.7)$ & $2(12.5)$ & $0(0.0)$ \\
\hline Three lobes & $0(0.0)$ & $1(6.3)$ & $0(0.0)$ \\
\hline More than three lobes & $5(38.5)$ & $12(75.0)$ & $3(100.0)$ \\
\hline \multicolumn{4}{|l|}{ Region of lobes } \\
\hline Upper & $6(46.1)$ & $14(87.5)$ & $3(100.0)$ \\
\hline Middle & $5(38.5)$ & $13(81.3)$ & $3(100.0)$ \\
\hline Lower & $11(84.6)$ & $15(93.7)$ & $3(100.0)$ \\
\hline \multicolumn{4}{|l|}{ Distribution } \\
\hline Anterior & $5(38.5)$ & $10(62.5)$ & $1(33.3)$ \\
\hline Posterior & $12(92.3)$ & $15(93.7)$ & $3(100.0)$ \\
\hline Peripheral & $13(100.0)$ & $16(100.0)$ & $3(100.0)$ \\
\hline Central & $0(0.0)$ & $1(6.3)$ & $0(0.0)$ \\
\hline \multicolumn{4}{|l|}{ Lesions } \\
\hline GGO only & $7(53.8)$ & $5(31.3)$ & $0(0.0)$ \\
\hline $\begin{array}{l}\text { GGO with reticular and/ } \\
\text { or interlobular septal } \\
\text { thickening }\end{array}$ & 1(7.7) & $2(12.5)$ & $0(0.0)$ \\
\hline $\begin{array}{l}\text { GGO with consolida- } \\
\text { tion }\end{array}$ & $6(46.1)$ & $8(50.0)$ & $3(100.0)$ \\
\hline Air bronchogram & $8(61.5)$ & $3(18.8)$ & $2(66.7)$ \\
\hline $\begin{array}{l}\text { Bronchial wall thicken- } \\
\text { ing }\end{array}$ & $1(7.7)$ & $6(37.6)$ & $1(33.3)$ \\
\hline Reticular shadow & $4(30.7)$ & $2(12.5)$ & $1(33.3)$ \\
\hline CT halo & $4(30.7)$ & $6(37.6)$ & $0(0.0)$ \\
\hline Reversed halo sign & $1(7.7)$ & $0(0.0)$ & $0(0.0)$ \\
\hline Pericardial & $0(0.0)$ & $0(0.0)$ & $0(0.0)$ \\
\hline Nodules & $0(0.0)$ & $1(6.3)$ & $0(0.0)$ \\
\hline Pleural effusion & $0(0.0)$ & $1(6.3)$ & $1(33.3)$ \\
\hline Lymphadenopathy & $0(0.0)$ & $0(0.0)$ & $0(0.0)$ \\
\hline
\end{tabular}

lung lobe and originated in the core area of the lobular or subpleural regions. In the early stage, GGO was the main manifestation, and interstitial thickening of the interlobular gradually appeared, showing reticular shadow changes in the ground glass shadow. However, the interlobular septal was rarely thickened, and thickening and traction bronchiectasis occurred during the disease progression or repair period. These CT findings are in line with the two studies $[10,11]$. On the other hand, Shi reported obvious cystic changes, which were not shown in our study [12]. The differences could be related to the individual differences and the severity of the illness. Notably, we did not find gravity distribution, tree-bud pattern, cavernous, or cystic airspaces in the CT images. The central interstitium is rarely affected except in serious patients. In addition, COVID-19 patients were not found to have combined

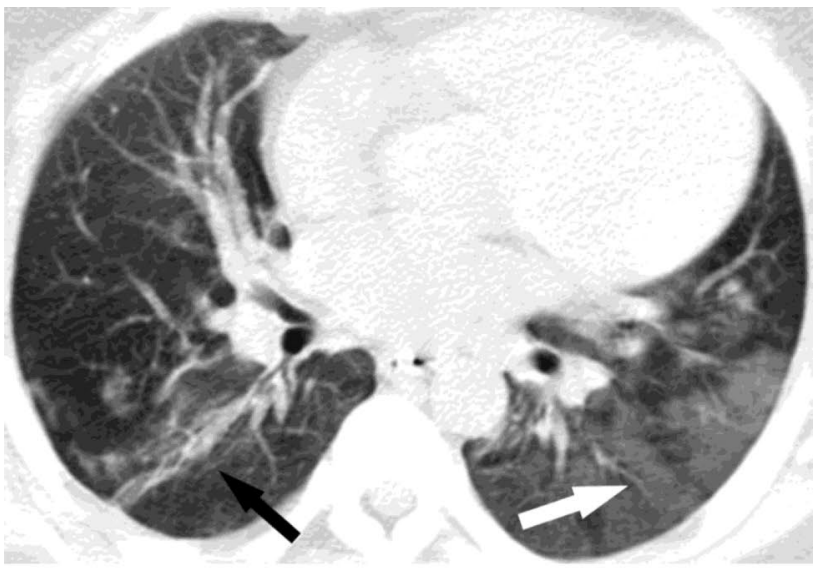

a

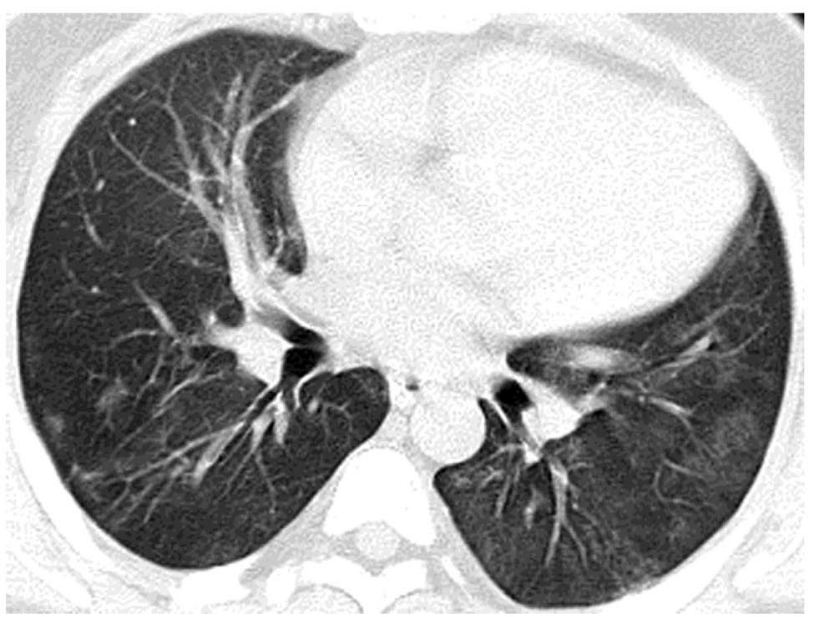

b

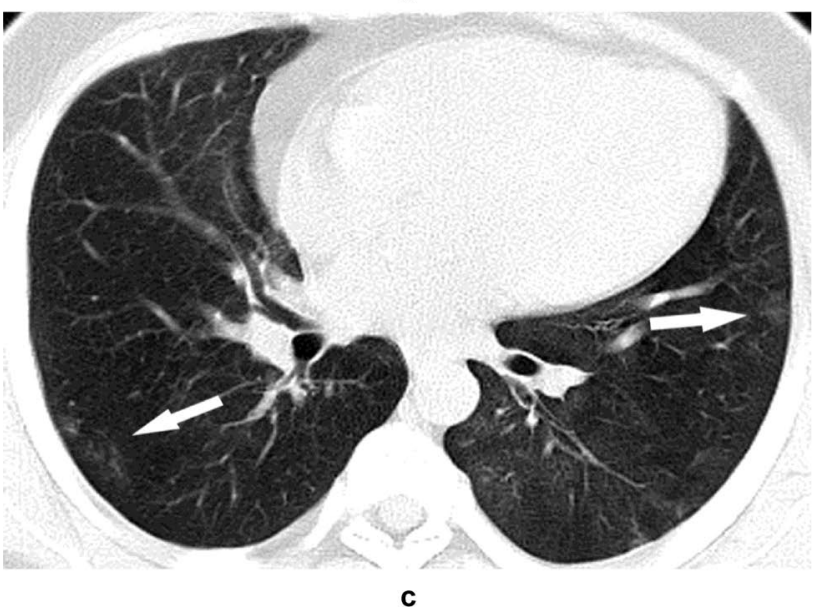

Fig. 2 Patient 1. Images in a 28-year-old male with fever and fatigue. a On the admission day, the unenhanced CT scan shows diffuse bilateral multiple patchy GGO (white arrow), and the partial boundary is clear while some have unclear boundaries, which are especially significant in the lower lobes of both lungs; strip consolidative opacities (black arrow) are in the focal area. b On day 5, the follow-up unenhanced CT scan shows a significant reduction in GGO in both lungs, and solid shadows have no new lesions. c On day 11, the follow-up unenhanced CT scan shows that the lesion was basically absorbed, and few GGOs remain (white arrows). On day 15, the patient was discharged from the hospital 


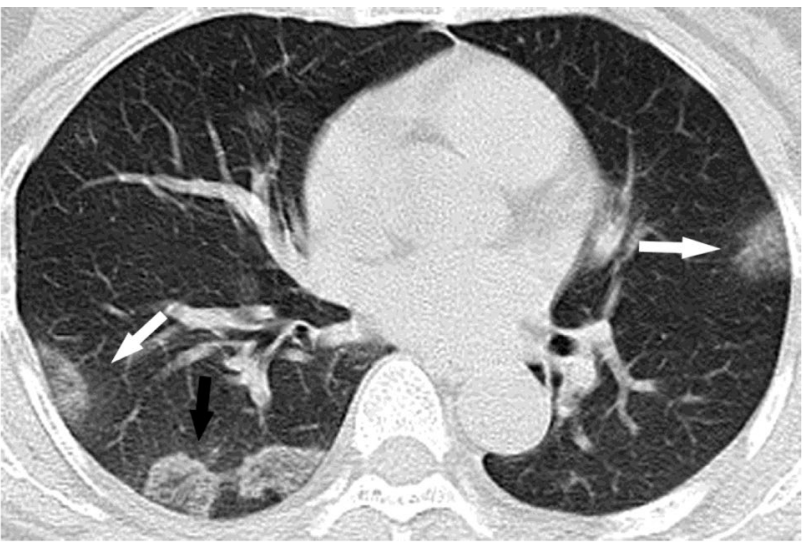

a

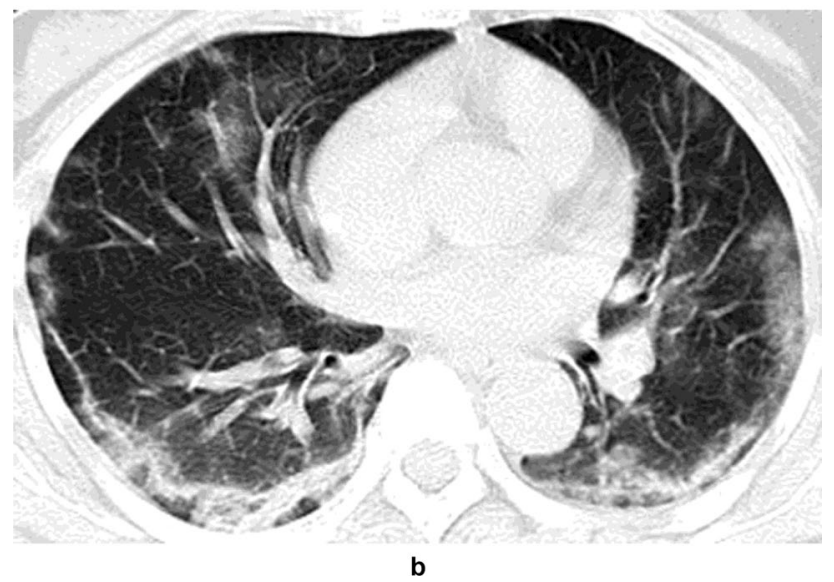

Fig. 3 Patient 4. Images in a 57-year-old woman with fever. The DR is normal in the lung on the admission day. a The unenhanced CT shows multiple patchy GGO-like lesions in the both lungs at subpleural position, unclear boundaries of GGO shadows with halo signs (white arrows) or clear boundaries of partial glass density shadows with reversed halo signs (black arrows). The mesh shadow is visible inside (black arrow). b Follow-up unenhanced CT on day 12 shows multiple new lesions. The lesions spread and fused in the subpleural area, showing a crescentic shape, while some lesions changed in consolidation and partial fibrosis

infections with bacteria and fungi in our studies and other studies [13]. These features could be considered key identification points from other viral pneumonia.

CT was indeed helpful to improve the clinical diagnosis of COVID-19 at an early stage. Patients 9, 19 and 25 were asymptomatic, but CT showed intrapulmonary lesions. Small thin and patchy GGO could be seen on the chest CT in patient 21 (Fig. 5), and the PCR results were negative twice. Later, the third PCR result was positive, and the lung had significant lesions with progression. A woman who is the wife of the patient 3 and mother of patient 22 had two negative PCR results, but the lesions in her lung had the same progression, and the blood test also confirmed the SARS-CoV-2 infection.

PCR of nasopharyngeal aspirate was regarded as a more sensitive method than conventional methods and was able to detect microorganisms that are difficult to culture and new microorganisms $[14,15]$. However, PCR as a diagnostic standard has the following problems. The shortage of PCR reagents and detectability delayed the diagnosis of SARS-CoV-2. Sometimes different brands of reagents would have different results. PCR tests should be collected and detected by experienced clinical technicians and detected by approved clinical laboratories, which limits its usage and is difficult to implement in basic hospitals. When the virus load was low in the early infection, it was hard to detect, and the potential for false negative results needs to be considered and carefully controlled in PCR experiments [16]. Most importantly, PCR cannot reflect the severity and progression of pneumonia, while CT imaging could support this vacancy. PCR tests showed false negative results in our study, which is consistent with previous studies $[10,11]$. In addition, since PCR results required a 1- to 3-day delay and then identified patients as confirmed cases, chest CT manifestations could appear earlier than viral nucleic acid assay in some cases. It is reasonable that $\mathrm{CT}$ could reflect the progression of the diseases and was of great importance in the early diagnosis and isolation of suspected patients.

The main weakness of our study was that we had only 25 patients, and our tracking time was very short. None of the patients were death cases, which limits our study. However, we focused on the first 25 patients in the Luoyang area and the early stage of the emerging SARS-CoV-2 infection, which had given information as a good reference for a quick response for other cities importing COVID-19 cases.

In summary, we suggested CT and epidemiological history as the primary clues and clinical symptoms and routine laboratory tests as the secondary clues for the early clinical diagnosis of suspicious patients to implement isolation and then wait for the nucleic acid results. For respiratory infectious diseases, regardless of viral pneumonia, it has high infectivity, and early isolation, diagnosis and treatment could effectively reduce the transmission rate and mortality of COVID-19 and control the global development of the epidemic. 
Fig. 4 Patient 10. Images in a 53-year-old man with fever. a On the admission day, the unenhanced CT scan shows multiple patchy GGO in the subpleural area of both lungs, localized consolidation, air bronchogram sign (white arrow), and slightly thickened localized vascular bundle (black arrow). b Follow-up unenhanced CT on day 6 shows a decrease in GGO density, an occurrence of new lesions on the subpleural and interlobular clefts, which partially combined to produce a patchy shape

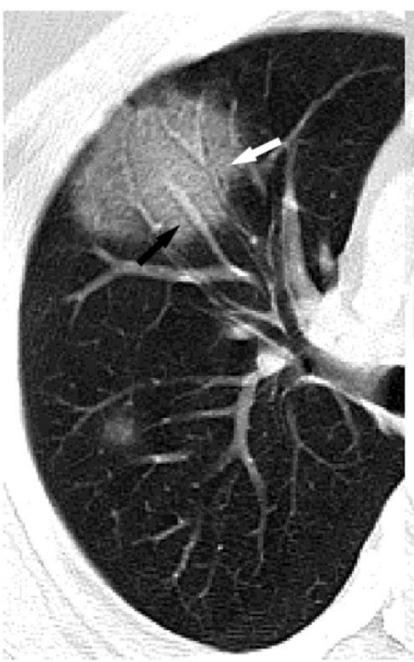

a

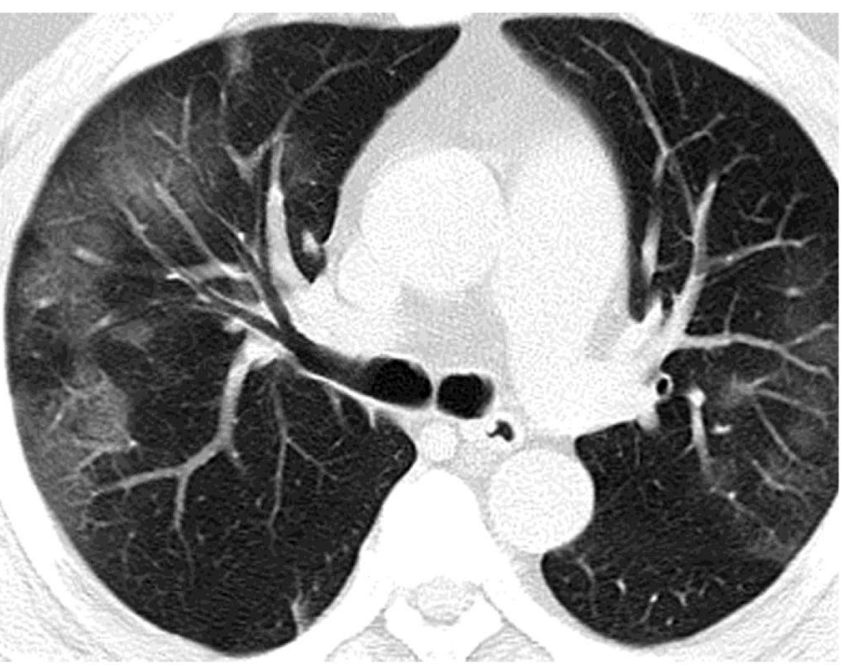

b

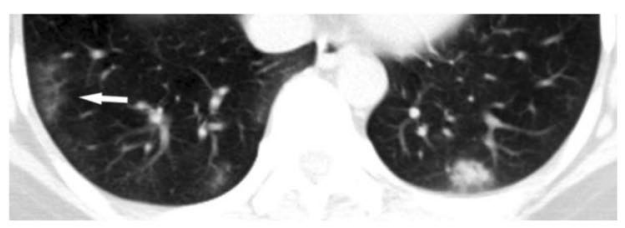

a

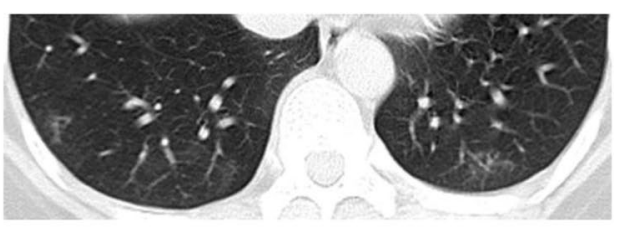

b
Fig. 5 Patient 22. Images in a 56-year-old man with fatigue. a The unenhanced CT scan shows a small patchy GGO shadow and small patchy consolidation in the subpleural area of both lungs. The boundary is unclear, and the capillaries increase (white arrow), whereas viral nucleic acid assay results were negative on this day and the next day. b Follow-up unenhanced CT on day 6 shows a decrease in the lesion density, with only small patchy thin GGOs and no new lesions. However, the third viral nucleic acid assay results were negative on the next day

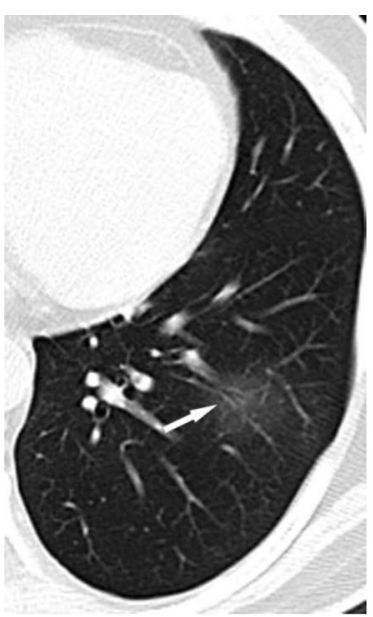

a

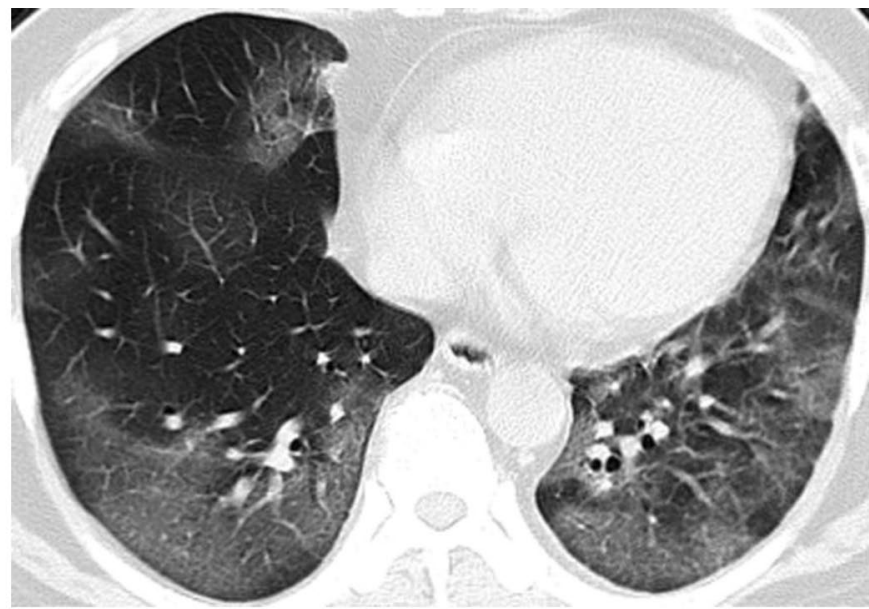

b
Fig. 6 Patient 22. Images in a 56-year-old man with fever. a The unenhanced CT scan shows a small patchy thin GGO shadow on the left inferior pulmonary lobe (white arrow) and localized bronchiole wall thickening. However, the viral nucleic acid assay results were reported to be negative on the next 2 consecutive days. b Follow-up unenhanced CT on day 10 shows multiple GGO lesions on the subpleural area in both lungs with partial consolidation, air bronchogram signs, and increased shadows in the capillaries. Then, viral nucleic acid assay results were reported to be positive on day 11 
Author contributions Guarantor of integrity of entire study: XD, JQ. Literature research: XD, XG. Clinical studies: XD, XG. Data acquisition: XD, XG. Data analysis/interpretation: XD, JQ. Statistical analysis: XG. Manuscript preparation: XG. Manuscript definition of intellectual content: XD, XG, JQ. Manuscript editing: XG. Manuscript revision: $X D, X G$. Manuscript final version approval: $X D, X G$. XD and $\mathrm{XG}$ contributed equally to the article.

\section{Compliance with ethical standards}

Conflict of interest The authors declare that they have no conflict of interest.

Ethical approval All procedures performed in studies involving human participants were in accordance with the ethical standards of The First Affiliated Hospital, College of Clinical Medicine, Medical College of Henan University of Science and Technology and with the 1964 Helsinki Declaration and its later amendments or comparable ethical standards.

Informed consent Informed consent was obtained from all individual participants included in the study.

\section{References}

1. Huang C, Wang Y, Li X, Ren L, Zhao J, Hu Y, et al. Clinical features of patients infected with 2019 novel coronavirus in Wuhan, China. Lancet. 2020;395(10223):497-506. https://doi. org/10.1016/S0140-6736(20)30183-5.

2. Li Q, Guan X, Wu P, Wang X, Zhou L, Tong Y, et al. Early transmission dynamics in Wuhan, China, of novel coronavirus-infected pneumonia. N Engl J Med. 2020;382(13):1199-207. https://doi. org/10.1056/NEJMoa2001316.

3. Lu R, Zhao X, Li J, Niu P, Yang B, Wu H, et al. Genomic characterisation and epidemiology of 2019 novel coronavirus: implications for virus origins and receptor binding. Lancet. 2020;395(10224):565-74. https://doi.org/10.1016/S0140 $-6736(20) 30251-8$.

4. Zhu N, Zhang D, Wang W, Li X, Bo Yang B, Song J, et al. A novel coronavirus from patients with pneumonia in China, 2019. N Engl J Med. 2020;382:727-33. https://doi.org/10.1056/NEJMoa2001 017.

5. Gorbalenya AE, Baker SC, Baric RS, de Groot RJ, Drosten C, et al. Severe acute respiratory syndrome-related coronavirusthe species and its viruses, a statement of the Coronavirus Study Group. BioRxiv. 2020. https://doi.org/10.1101/2020.02.07.93786 2.
6. Chan JF, Yuan S, Kok KH, To KK, Chu H, Yang J, et al. A familial cluster of pneumonia associated with the 2019 novel coronavirus indicating person-to-person transmission: a study of a family cluster. Lancet. 2020;395(10223):514-23. https://doi.org/10.1016/ S0140-6736(20)30154-9.

7. Chen J. Pathogenicity and transmissibility of 2019-nCoV-A quick overview and comparison with other emerging viruses. Microbes Infect. 2020;22(2):69-71. https://doi.org/10.1016/j. micinf.2020.01.004.

8. WHO. Clinical management of severe acute respiratory infection when novel coronavirus (nCoV) infection is suspected. https:// www.who.int/publications-detail/clinical-management-of-sever e-acute-respiratory-infection-when-novel-coronavirus-(ncov)infection-is-suspected (2020). Accessed 28 Jan 2020

9. Guan W, Ni Z, Hu Y, Liang W, Ou C, He J, et al. Clinical characteristics of 2019 novel coronavirus infection in China. MedRxiv. 2020. https://doi.org/10.1101/2020.02.06.20020974.

10. Xie X, Zhong Z, Zhao W, Zheng C, Wang F, Liu J. Chest CT for typical 2019-nCoV pneumonia: relationship to negative RT-PCR testing. Radiology. 2020;12:200343. https://doi.org/10.1148/radio 1.2020200343.

11. Fang Y, Zhang H, Xie J, Lin M, Ying L, Pang P, Ji W. Sensitivity of chest CT for COVID-19: comparison to RT-PCR. Radiology. 2020. https://doi.org/10.1148/radiol.2020200432.

12. Shi H, Han X, Jiang N, Cao Y, Alwalid O, Gu J, et al. Radiological findings from 81 patients with COVID-19 pneumonia in Wuhan, China: a descriptive study. Lancet Infect Dis. 2020;20(4):425-34. https://doi.org/10.1016/S1473-3099(20)30086-4.

13. Koo HJ, Lim S, Choe J, Choi SH, Sung H, Do KH. Radiographic and CT features of viral pneumonia. Radiographics. 2018;38(3):719-39. https://doi.org/10.1148/rg.2018170048.

14. Munywoki PK, Hamid F, Mutunga M, Welch S, Cane P, Nokes DJ. Improved detection of respiratory viruses in pediatric outpatients with acute respiratory illness by real-time PCR using nasopharyngeal flocked swabs. J Clin Microbiol. 2011;49(9):3365-7. https:// doi.org/10.1128/JCM.02231-10.

15. van de Pol AC, van Loon AM, Wolfs TF, Jansen NJ, Nijhuis M, Breteler EK, et al. Increased detection of respiratory syncytial virus, influenza viruses, parainfluenza viruses, and adenoviruses with real-time PCR in samples from patients with respiratory symptoms. J Clin Microbiol. 2007;45(7):2260-2. https://doi. org/10.1128/JCM.00848-07.

16. Bacich DJ, Sobek KM, Cummings JL, Atwood AA, O'Keefe DS. False negative results from using common PCR reagents. BMC Res Notes. 2011;4:457. https://doi.org/10.1186/1756-0500-4-457.

Publisher's Note Springer Nature remains neutral with regard to jurisdictional claims in published maps and institutional affiliations. 